\title{
Dendritic cell vaccine and cytokine-induced killer cell therapy for the treatment of advanced non-small cell lung cancer
}

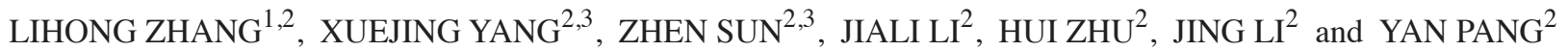 \\ ${ }^{1}$ School of Medicine, Nankai University, Tianjin 300071; ${ }^{2}$ Department of Oncology, Tianjin Union Medicine Centre, \\ Tianjin 300121; ${ }^{3}$ Shanghai Claison Biotechnology Co., Ltd., Shanghai 201201, P.R. China
}

Received February 3, 2015; Accepted January 26, 2016

DOI: $10.3892 / \mathrm{ol} .2016 .4273$

\begin{abstract}
The present study aimed to evaluate the survival time, immune response and safety of a dendritic cell (DC) vaccine and cytokine-induced killer (CIK) cell therapy (DC-CIK) in advanced non-small cell lung cancer (NSCLC). The present retrospective study enrolled 507 patients with advanced NSCLC; 99 patients received DC-CIK [immunotherapy group (group I)] and 408 matched patients did not receive DC-CIK, and acted as the control [non-immunotherapy group (group NI)]. Delayed-type hypersensitivity (DTH), quality of life (QOL) and safety were analyzed in group I. The follow-up period for the two groups was $489.2 \pm 160.4$ days. The overall survival (OS) time was calculated using the Kaplan-Meier method. DTH was observed in 59 out of 97 evaluated patients (60.8\%) and 67 out of 98 evaluated patients (68.4\%) possessed an improved QOL. Fever and a skin rash occurred in 36 out of 98 patients $(36.7 \%)$ and 7 out of 98 patients $(7.1 \%)$ in group I. DTH occurred more frequently in patients with squamous cell carcinoma compared with patients with adenocarcinoma (77.1 vs. $40.4 \%$; $\mathrm{P}=0.0013$ ). Radiotherapy was not associated with DC-CIK-induced DTH (72.7 vs. 79.6\%; P=0.18), but chemotherapy significantly reduced the rate of DTH (18.2 vs. $79.6 \%$; $\mathrm{P}=0.00)$. The $\mathrm{OS}$ time was significantly increased in group I compared with group NI $(\mathrm{P}=0.03)$. In conclusion, DC-CIK may induce an immune response against NSCLC, improve the QOL, and prolong the OS time of patients, without adverse effects. Therefore, the present study recommends DC-CIK for the treatment of patients with advanced NSCLC.
\end{abstract}

\section{Introduction}

Lung cancer is the leading cause of cancer-associated mortality and the most common cancer type worldwide (1). In

Correspondence to: Professor Yan Pang, Department of Oncology, Tianjin Union Medicine Centre, 190 Jieyuan Road, Tianjin 300121, P.R. China

E-mail: pangyancn@126.com

Key words: dendritic cell vaccine, cytokine-induced killer cells, non-small cell lung cancer, immunotherapy total, $\sim 80 \%$ of all lung cancer cases are non-small cell lung cancer (NSCLC) (2), for which surgery, chemotherapy and radiotherapy remain the standard treatments (3). The majority of patients with NSCLC are not recommended for surgery, since patients are often diagnosed at an advanced stage of disease (stage IIIb or IV). Patients that do undergo radical surgery may eventually develop locoregional recurrence or distant metastasis (4). Characterized by inexorable disease progression, advanced NSCLC usually has a high malignant potential. Its prognosis is poor and the clinical onset is extensive, despite treatment with chemotherapy and radiation (5). Systemic chemotherapy is the recommended first-line treatment for patients with advanced stage and metastatic NSCLC, but it is often considered ineffective or excessively toxic (6).

Multidisciplinary approaches are required to develop novel treatments for advanced NSCLC (7). One of these multidisciplinary approaches is immunotherapy $(7,8)$, an important and effective method in cancer treatment, particularly for advanced-stage disease (9). Immune cells, including cytokine-induced killer (CIK) cells and dendritic cells (DCs), aid in mounting an effective immune response against cancer cells and kill cancer cells, including residual cells (10-13). A combination of conventional methods, such as surgery, chemotherapy and radiotherapy, combined with immunotherapy is a novel approach to reducing mortality from cancer $(3,14)$.

In the present study, the immune response induced by a DC vaccine and CIK cell therapy (DC-CIK), the adverse effects of the vaccine, and improvements in the quality of life (QOL) and overall survival (OS) time were evaluated in patients with advanced NSCLC.

\section{Patients and methods}

Patients and immunotherapy. All patients were enrolled at the Department of Oncology, Tianjin Union Medicine Centre (Tianjin, China). The present study evaluated two groups of patients, consisting of patients that received DC-CIK [immunotherapy group (group I)] and matched patients that did not receive DC-CIK [non-immunotherapy group (group NI)], who acted as the control. Group I consisted of hospitalized patients with NSCLC that underwent DC-CIK between January 2012 and October 2012, and who possessed stage IIIb or IV disease and locoregional recurrence or distant metastasis following surgery for NSCLC. The immunotherapy 
patients underwent blood tests and met the following requirements: Lymphyocytes and monocytes, $>0.7 \times 10^{9} / 1$; white blood cells, $>3.0 \times 10^{9}$ cells $/ 1$; hemoglobin, $>75.0 \mathrm{~g} / \mathrm{l}$; platelets, $>50.0 \times 10^{9}$ platelets $/ 1$; coagulation: prothrombin time, $<22.5 \mathrm{sec}$; activated partial thromboplastin time, $<65 \mathrm{sec}$; thrombin time, $<32 \mathrm{sec}$; cardiac function, $\leq$ III level (15); liver function, <Child-Pugh Class/Score C (16); no renal failure or uremia. The requirements indicated adequate kidney, liver, coagulation and bone marrow function. Group NI consisted of NSCLC patients with matched characteristics and treatments to the patients in group I, but did not receive DC-CIK.

The present study was performed at the Department of Oncology of Tianjin Union Medicine Centre (Tianjin, China). Following the policy outlined by the Ministry of Health of China, the present study was approved as a class III medical technique, and was listed in Treatment with Autologous Immune Cells (T cells, NK cells) $(17,18)$. All patients provided written informed consent prior to treatment, as required by the Ethics Committee of Tianjin Union Medicine Centre. The present study was a retrospective study using data obtained from the medical records of patients that received a standard treatment for NSCLC. The clinical data were collected with permission from the Inpatients Electronic Medical Records of Tianjin Union Medicine Centre.

Preparation of DCs and CIK cells. On the first day of treatment, leukocyte fractions were collected from the patients via leukapheresis using the Fresenius KABI System (COM.TEC; Fresenius SE \& Co. KGaA, Homburg, Germany) under electrocardiographic monitoring. Peripheral blood mononuclear cells (PBMCs) were isolated via Ficoll-Hypaque gradient centrifugation and cultured for $2 \mathrm{~h}$ [DCs, RPMI-1640 (Gibco ${ }^{\circledR}$; Thermo Fisher Scientific, Inc., Waltham, MA, USA); CIK, GT-T551 medium (Takara Bio, Inc., Beijing China)] in a Forma ${ }^{\mathrm{TM}}$ Series II $3111 \mathrm{CO}_{2}$ Waterjacket Incubator (Thermo Fisher Scientific, Inc.). The human lung squamous cell carcinoma SK-MES-1 and human lung adenocarcinoma SPC-A-1 cell lines (Chinese Academy of Sciences, Shanghai, China) were cultured in Gibco Cell Culture Media (Thermo Fisher Scientific, Inc.) for $24 \mathrm{~h}$ at $37^{\circ} \mathrm{C}(19-21)$. The cells were lysed by ultrasound and centrifuged (Heraeus Fresco 17; Thermo Fisher Scientific, Inc.) at $600 \mathrm{x} \mathrm{g}$ for $30 \mathrm{~min}$. The supernatants were collected and used as a tumor lysate for DC preparation. DCs and CIK cells were prepared as described in a previous study (22-24). The supernatants of cultured PBMCs were removed for the additional preparation of CIK cells and the remaining cells were cultured for 7 days in the presence of granulocyte-macrophage colony stimulating factor, interleukin (IL)-4 and tumor necrosis factor (PeproTech EC, Ltd., London, UK), and pulsed with the tumor lysate (PeproTech EC, Ltd.) (25). On the ninth day, the DCs were harvested for immediate vaccinations $(12,26)$ or frozen for subsequent reinfusion. The supernatants were washed, and were cultured in the presence of interferon- $\gamma$, rat anti-human anti-cluster of differentiation 3 monoclonal antibody (catalog no., T210; dilution, 1:100; Takara Bio, Inc.) and IL-2 (PeproTech EC, Ltd.) (27). The cells were harvested between days 13 and 15 .

Clinical regimen. The viability of DCs and CIK cells was $>70$ and $90 \%$, respectively, detected using flow cytometry
(FC500; Beckman Coulter, Miami, FL, USA). The cells were subjected to a bacterial gram stain, bacterial culture, fungal culture and bacterial endotoxin test to ensure they were not contaminated with bacteria, fungi or mycoplasma, and endotoxin levels in the cells were $>5 \mathrm{EU} / \mathrm{kg}$ prior to infusion. On the ninth day, $1 \times 10^{7}$ vaccine DCs suspended in $100 \mathrm{ml}$ normal saline (NS) were infused intravenously once a week for 3 weeks. Subsequently, $1.0 \times 10^{7} \mathrm{DCs}$ suspended in $4 \mathrm{ml} \mathrm{NS}$ in 2 syringes were infused intradermally once a week for an additional 3 weeks. On the twelfth day, $1 \times 10^{9} \mathrm{CIK}$ cells suspended in $100 \mathrm{ml} \mathrm{NS}$ were infused intravenously once a day for 4 consecutive days.

Immune response. In total, 1 week subsequent to the last DC vaccination, delayed-type hypersensitivity (DTH) of the patients was tested as an immune response marker. A total of $1 \times 10^{7}$ DCs in $0.1 \mathrm{ml} \mathrm{NS}$ was administrated intradermally into the forearm of each patient. The patients were asked to measure the diameter of the skin erythema around the injection point following 24, 48 and $72 \mathrm{~h}$. The DTH reaction was defined as follows: $>10 \mathrm{~mm}$ in diameter, strongly positive; $5-10 \mathrm{~mm}$, positive; $2-5 \mathrm{~mm}$, weakly positive; and $<2 \mathrm{~mm}$, negative $(22,28)$.

$Q O L$. The QOL of the patients was evaluated based on physical strength, appetite, amount of sleep and body weight (29). The patients were provided a clinical response questionnaire, on which they recorded feedback regarding the effect of the treatment. QOL score was determined by the number of improved factors, ranging between 0 , indicating no improvement, and 4 , indicating all factors improved.

Safety. Fever was the most common adverse effect of DC-CIK and was of considerable concern. Fever was defined as a cold-like symptom, with varying temperatures defining the severity of the fever, as follows: $<38^{\circ} \mathrm{C}$, Low fever; $38-39^{\circ} \mathrm{C}$, moderate fever; and $>39^{\circ} \mathrm{C}$, high fever. Skin rashes were also observed during therapy.

Adverse effects. Adverse reactions, including insomnia, anorexia and joint soreness were monitored during DC-CIK. A slight increase in body temperature was regarded as a cold-like symptom. Fever, skin rashes and other symptoms previously observed during DC-CIK were recorded (22). Adverse effects known to be associated with chemotherapy and radiotherapy, such as nausea, vomiting, bone marrow suppression and organ function impairment, including the kidneys, liver, lungs and heart, were also monitored.

OS time. The OS time was calculated as the date from which the patients were enrolled in the present study to the date that the patient succumbed to NSCLC or any other cause. The OS time for the two groups was analyzed and compared using Kaplan-Meier estimates.

Data collection and statistical analysis. The primary end-points for the present study were the immune response and QOL of the patients, and the safety of the therapy. The secondary end-point was the OS time of the patients (30). Clinical data were collected using EpiData software version 3.02 (EpiData Association, Odense, Denmark). Statistical analyses were performed using SPSS software version 19.0 (IBM SPSS, 
Table I. Characteristics of patients with advanced non-small cell lung cancer.

\begin{tabular}{|c|c|c|c|c|}
\hline Characteristics & Group I, n (\%) & Group NI, n (\%) & Total, n (\%) & P-value \\
\hline Total & $99(100.0)$ & $408(100.0)$ & $507(100.0)$ & \\
\hline Age, years & & & & 0.39 \\
\hline Range & $31-87$ & $29-93$ & $29-93$ & \\
\hline Mean \pm SD & $64.7 \pm 10.4$ & $68.1 \pm 10.6$ & $66.28 \pm 8.9$ & \\
\hline Gender & & & & 0.33 \\
\hline Male & $65(65.7)$ & $246(60.3)$ & $311(61.3)$ & \\
\hline Female & $34(34.3)$ & $162(39.7)$ & $196(38.7)$ & \\
\hline Degree of differentiation ${ }^{a}$ & & & & 0.99 \\
\hline High & $7(7.1)$ & $29(7.1)$ & $36(7.1)$ & \\
\hline Middle & $40(40.4)$ & $167(40.9)$ & $207(40.8)$ & \\
\hline Low & $52(52.5)$ & $211(51.7)$ & $263(51.9)$ & \\
\hline \multicolumn{5}{|l|}{ Tumor types } \\
\hline Squamous cell carcinoma & $35(35.4)$ & $184(45.1)$ & $219(43.2)$ & 0.21 \\
\hline Adenocarcinoma & $57(57.6)$ & $199(48.7)$ & $256(50.5)$ & \\
\hline Undefined & $7(7.1)$ & $25(6.1)$ & $32(6.3)$ & \\
\hline \multicolumn{5}{|l|}{ Combined therapies } \\
\hline Surgery & $11(11.1)$ & $33(8.1)$ & $44(8.7)$ & 0.34 \\
\hline Radiotherapy & $25(25.3)$ & $71(17.4)$ & $96(18.9)$ & 0.07 \\
\hline Chemotherapy & $25(25.3)$ & $82(20.1)$ & $107(21.1)$ & 0.26 \\
\hline
\end{tabular}

${ }^{a}$ One patient was classified with a middle-high degree of differentiation and was, therefore, excluded. Group I, immunotherapy group; Group NI, non-immunotherapy group; SD, standard deviation.

Table II. Grade of DTH, fever, allergy and QOL in patients with advanced non-small cell lung cancer treated with dendritic cell vaccine and cytokine-induced killer cell therapy.

\begin{tabular}{|c|c|c|c|c|}
\hline Response grade & DTH, n (\%) & Fever, n (\%) & Allergy, n (\%) & QOL, n (\%) \\
\hline Total & $97(98.0)$ & $98(99.0)$ & $98(99.0)$ & $98(99.0)$ \\
\hline Data loss & $2(2.0)$ & $1(1.0)$ & $1(1.0)$ & $1(1.0)$ \\
\hline \multicolumn{5}{|l|}{ Grade } \\
\hline 0 & $38(39.2)$ & $62(63.3)$ & $91(92.9)$ & $31(31.6)$ \\
\hline 1 & $24(24.7)$ & $14(14.3)$ & $5(5.1)$ & $26(26.5)$ \\
\hline 2 & $25(25.8)$ & $16(16.3)$ & $2(2.0)$ & $34(34.7)$ \\
\hline 3 & $10(10.3)$ & $6(6.1)$ & - & $7(7.1)$ \\
\hline
\end{tabular}

The DTH grade was characterized as: $0,<2 \mathrm{~mm} ; 1,2-5 \mathrm{~mm} ; 2,5-10 \mathrm{~mm}$; and 3,>10 mm. The fever grade was characterized as: 0 , normal; 1 , common cold-like symptoms and temperature $<38^{\circ} \mathrm{C} ; 2,38-39^{\circ} \mathrm{C}$; and $3,>39^{\circ} \mathrm{C}$. The allergy grade was characterized as: 0 , normal; 1, local erythema around injection point; and 2, systemic skin rash. The QOL was graded as: 0 , no improvement; and 1-3, improvement in 1,2-3 or 4 of the QOL factors, respectively. DTH, delayed-type hypersensitivity; QOL, quality of life.

Armonk, NY, USA). The Student's $t$-test was used for measurement data and the $\chi^{2}$ test was used for count data. The OS curves were calculated using the Kaplan-Meier method. $\mathrm{P}<0.05$ was considered to indicate a statistically significant difference.

\section{Results}

Patient characteristics. A total of 507 patients with advanced NSCLC were enrolled in the present study, consisting of
311 male and 196 female patients, with a mean age of 66.28 years (range, 29-93 years). The tumor diagnoses of the patients were as follows: Squamous cell carcinoma, 219 patients; adenocarcinoma, 256 patients; and undefined, 32 patients. The degree of NSCLC differentiation was high in 36 patients, medium in 207 patients and low in 263 patients. Among the 507 patients, $44(8.7 \%)$ underwent primary tumor resection, $96(18.9 \%)$ received radiotherapy and $107(21.1 \%)$ received chemotherapy within 3 months prior to the start of immunotherapy (Table I). 
Table III. Effect of radiotherapy and chemotherapy on DTH in patients with advanced non-small cell lung cancer that underwent DC-CIK.

\begin{tabular}{|c|c|c|c|c|c|}
\hline Groups & Total, $\mathrm{n}$ & $\mathrm{DTH}^{+}, \mathrm{n}$ & $\mathrm{DTH}^{-}, \mathrm{n}$ & Incidence of DTH, \% & P-value \\
\hline Total, $\mathrm{n}$ & 93 & 57 & 36 & 61.3 & \\
\hline Radiotherapy + DC-CIK & 22 & 16 & 6 & 72.7 & 0.213 \\
\hline Chemotherapy + DC-CIK & 22 & 4 & 18 & 18.2 & 0.001 \\
\hline DC-CIK & 49 & 39 & 10 & 79.6 & - \\
\hline
\end{tabular}

In total, 3 patients were excluded from the analysis since they received radiotherapy and chemotherapy concurrently. P-value vs. dendritic cell vaccine and cytokine-induced killer cell therapy. DTH, delayed-type hypersensitivity; DC-CIK, dendritic cell vaccine and cytokine-induced killer cell therapy.

Table IV. Association between DTH and tumor type in patients with advanced non-small cell lung cancer that underwent dendritic cell vaccine and cytokine-induced killer cell therapy therapy.

\begin{tabular}{lcccc}
\hline Tumor types & Total, $\mathrm{n}$ & $\mathrm{DTH}^{+}, \mathrm{n}$ & $\mathrm{DTH}^{-}, \mathrm{n}$ & Incidence of DTH, \% \\
\hline Total, $\mathrm{n}$ & 92 & 50 & 42 & 54.3 \\
Squamous cell carcinoma & 35 & 27 & 8 & 77.1 \\
Adenocarcinoma & 57 & 23 & 34 & 40.4 \\
\hline
\end{tabular}

DTH, delayed-type hypersensitivity; DC-CIK, dendritic cell vaccine and cytokine-induced killer cell therapy.

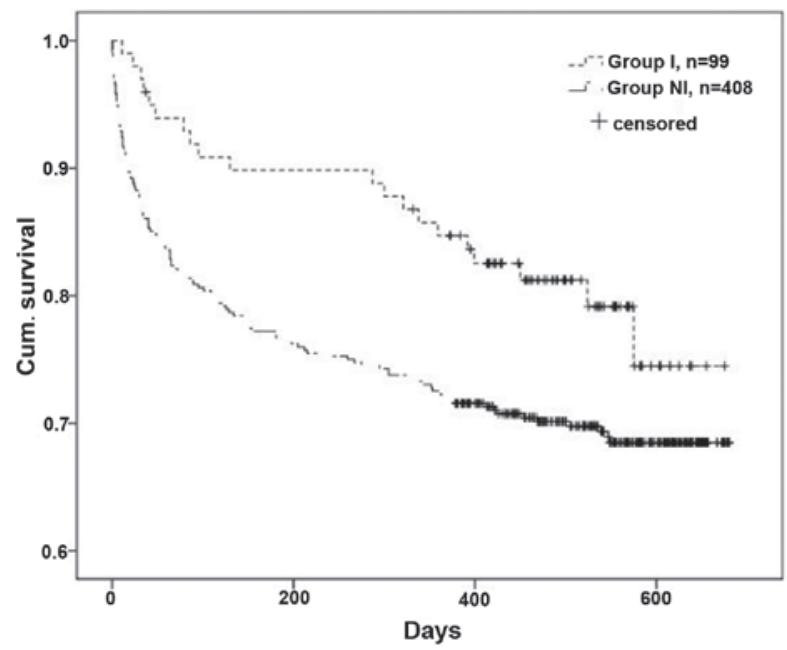

Figure 1. Overall survival curves were calculated with the Kaplan-Meier method for patients in the two groups. In total, 20 out of 99 patients in group I and 145 out of 408 patients in group NI succumbed to NSCLC or other causes $(\mathrm{P}=0.03)$. Group I, immunotherapy group; Group NI, non-immunotherapy group; Cum., cumulative.

The 507 patients were divided into two groups. A total of 408 patients that received a routine treatment regimen consisting of surgery, radiotherapy and chemotherapy were enrolled into the non-immunotherapy group (control group; group NI). In addition, 99 patients that received DC-CIK in addition to the routine treatments were classified as the immunotherapy group (group I). The characteristics of the patients, including age, gender, diagnostic methods and tumor type, were similar between the two groups. With the exception of the use of DC-CIK, the treatment strategies did not differ significantly between the two groups (Table I).

DTH skin test. The DTH skin test results were available for 97 out of the 99 patients in group I, of whom $59(60.8 \%)$ possessed a positive result. In total, 10 of these patients $(10.3 \%)$ had a strongly positive result; $25(25.8 \%)$ had a positive result; $24(24.7 \%)$ had a weakly positive result; and 38 (39.2\%) had a negative result (Table II). The results from 2 patients were unknown.

QOL. The 4 QOL factors assessed were physical strength, appetite, amount of sleep and body weight. QOL scores were available for 98 out of the 99 patients in group I, as follows: 7 patients $(7.1 \%)$ demonstrated improvements in all 4 QOL factors; 34 patients $(34.7 \%)$ demonstrated improvements in 2-3 factors; and 26 patients $(26.5 \%)$ demonstrated improvements in a single factor. Overall, $68.4 \%$ of the group I patients (67 out of 98) possessed an improved QOL following immunotherapy, while the remaining $31.6 \%$ of patients (31 out of 98) had no improvement in QOL (Table II).

Adverse effects. Body temperature was recorded in 98 out of the 99 patients in group I. In these 98 patients, cold-like symptoms with a low fever were observed in 14 patients $(14.3 \%)$. A moderate fever was observed in 16 patients $(16.3 \%)$ and a high fever was observed in 6 patients $(6.1 \%)$. In total, 36 of the 98 patients $(36.7 \%)$ experienced fever, including cold-like symptoms. No fever was detected in the remaining 62 patients $(63.3 \%)$ during DC-CIK (Table II). The presence or absence of allergy was recorded in 7 of the 98 patients in group I, 2 of whom experienced a skin rash $(2.0 \%)$ and 5 of whom 
experienced local erythema around the injection site. No additional toxicities were observed in patients receiving DC-CIK (Table II) (31).

Association between DTH and tumor type. In group I, 27 of the 35 patients $(77.1 \%)$ with squamous cell carcinoma of the lung developed DTH. By contrast, 23 of the 57 patients $(40.4 \%)$ with adenocarcinoma of the lung developed DTH $(\mathrm{P}=0.0013$; Table IV).

Effect of radiotherapy and chemotherapy on DTH. The association between radiotherapy, chemotherapy and DTH was assessed in 93 out of the 96 patients with NSCLC in group I. The remaining 3 patients received radiotherapy and chemotherapy concurrently and were excluded from the analysis.

There was no significant difference between the rate of DTH in the 49 patients in group I that did not undergo radiotherapy $(79.6 \%)$ compared with the 22 patients that did (72.7\%). However, the incidence of DTH was significantly lower amongst the patients that underwent chemotherapy (18.2\%) compared with those patients that did not $(79.6 \%$; $\mathrm{P}=0.22$; Table III).

OS time. The OS time of patients in group I was estimated and compared to the OS time of patients in group NI using the Kaplan-Meier method. In total, 20 out of 99 patients $(20.2 \%)$ in group I and 125 out of 408 patients (30.6\%) in group NI succumbed to NSCLC or other causes during the present study. The OS time of patients in group I was significantly improved compared with group NI ( $\mathrm{P}=0.03$; Fig. 1) (32).

\section{Discussion}

Despite rapid advances in surgery, radiotherapy and chemotherapy, which are effective modalities in cancer treatment, the prognosis following treatment for NSCLC remains poor (33). To improve the clinical outcome for patients with NSCLC, novel treatment modalities are urgently required. One such alternative is immunotherapy with DC-CIK, where DCs have previously been revealed to activate CIK cells, which enhances antitumor effects in NSCLC patients $(34,35)$. An adoptive transfer of CIK cells with DCs has proven to be an effective strategy for extending the OS time in patients $(7,36)$, enhancing host cellular immune responses and improving the QOL of patients $(8,37)$. Furthermore, no severe adverse effects were reported during DC-CIK cell transfusion, indicating that this therapy is safe for clinical use. Adverse effects were mild and were effectively managed.

The present study used the DTH skin test to assess the cell-mediated cytotoxicity response induced by DC-CIK, and identified that $61 \%$ of DC-CIK-treated patients (59 out of 97) had a positive reaction. In addition, $68 \%$ of patients (67 out of 98) demonstrated improved physical strength, appetite, sleep and body weight. No toxicity other than fever and skin rash was associated with DC-CIK. Fever was observed in 26 of the 98 patients (27\%) in group I and was generally mild and temporary. During these episodes, body temperature was rarely $>39^{\circ} \mathrm{C}$ and was relieved by cooling or antipyretic analgesics. Cold-like symptoms, regarded as a mild form of fever, were observed in 14 out of the 98 patients (14\%). In these cases, patients complained of symptoms, including fatigue and sore joints, but their temperatures were normal $\left(36-37^{\circ} \mathrm{C}\right)$. Allergic reactions, indicated by a skin rash, were rare, occurring in only 7 out of the 98 patients (7.1\%), and usually mild and self-resolving without requiring special treatments (Table II). The rate of DTH in patient with squamous cell carcinoma of the lung was significantly higher compared with patients with adenocarcinoma of the lung (77.1 vs. $40.4 \%$; $\mathrm{P}=0.0013$; Table IV). Radiotherapy had no effect on DTH induced by DC-CIK (72.7 vs. 79.6\%; $\mathrm{P}=0.02$ ), but chemotherapy significantly reduced the incidence of DTH (18.2 vs. $79.6 \%$; $\mathrm{P}=0.001$; Table III).

The OS time of group I patients was estimated and compared to the OS time of group NI patients using the Kaplan-Meier method. In total, 20 out of the 99 patients in group I, and 125 out of the 408 patients in group NI succumbed to NSCLC or other causes during the present study. The improvement in the OS time of patients in group I was significant $(\mathrm{P}=0.03$; Fig. 1) (11).

The present findings suggest that immunotherapy with DC-CIK has antitumor effects and, in combination with conventional treatment therapies, may prolong the OS time of advanced NSCLC patients $(32,34)$.

Severe treatment-associated adverse effects and inadequate outcomes associated with conventional treatments may account for the poor prognosis of patients with advanced NSCLC. The present study revealed that there were no severe adverse effects resulting from DC-CIK therapy. Furthermore, this therapy mounted an immune response against NSCLC, and consequently lengthened the OS time and improved the QOL of patients (35). The present study concludes that DC-CIK may be beneficial for patients with advanced NSCLC.

\section{Acknowledgements}

The present study was partially supported by the Tianjin Municipal Science and Technology Commission (grant no., 12ZCDZSY17100).

\section{References}

1. Gupta N, Hatoum H and Dy GK: First line treatment of advanced non-small-cell lung cancer - specific focus on albumin bound paclitaxel. Int J Nanomedicine 9: 209-221, 2014.

2. Zhong R, Han B and Zhong H: A prospective study of the efficacy of a combination of autologous dendritic cells, cytokine-induced killer cells, and chemotherapy in advanced non-small cell lung cancer patients. Tumour Biol 35: 987-994, 2014.

3. Zarogoulidis K, Zarogoulidis P, Darwiche K, Boutsikou E, Machairiotis N, Tsakiridis K, Katsikogiannis N, Kougioumtzi I, Karapantzos I, Huang H and Spyratos D: Treatment of non-small cell lung cancer (NSCLC). J Thorac Dis 5 (Suppl 4): S389-S396, 2013.

4. Hirsh V: Review of the treatment of metastatic non small cell lung carcinoma: A practical approach. World J Clin Oncol 2: 262-271, 2011.

5. Fiala O, Pesek M, Finek J, Svaton M, Sorejs O, Bortlicek Z, Kucera R and Topolcan O: Prognostic significance of serum tumor markers in patients with advanced-stage NSCLC treated with pemetrexed-based chemotherapy. Anticancer Res 36: 461-466, 2016.

6. Choi MK, Hong JY, Chang W, Kim M, Kim S, Jung HA, Lee SJ, Park S, Chung MP, Sun JM, et al: Safety and efficacy of gemcitabine or pemetrexed in combination with a platinum in patients with non-small-cell lung cancer and prior interstitial lung disease. Cancer Chemother Pharmacol 73: 1217-1225, 2014. 
7. Yang L, Ren B, Li H, Yu J, Cao S, Hao X and Ren X: Enhanced antitumor effects of DC-activated CIKs to chemotherapy treatment in a single cohort of advanced non-small-cell lung cancer patients. Cancer Immunol Immunother 62: 65-73, 2013.

8. Kelly RJ, Gulley JL and Giaccone G: Targeting the immune system in non-small-cell lung cancer: Bridging the gap between promising concept and therapeutic reality. Clin Lung Cancer 11: 228-237, 2010

9. Aktipis CA and Nesse RM: Evolutionary foundations for cancer biology. Evol Appl 6: 144-159, 2013.

10. Schürch CM, Riether C and Ochsenbein AF: Dendritic cell-based immunotherapy for myeloid leukemias. Front Immunol 4: 496, 2013.

11. Liu H, Song J, Yang Z and Zhang X: Effects of cytokine-induced killer cell treatment combined with FOLFOX4 on the recurrence and survival rates for gastric cancer following surgery. Exp Ther Med 6: 953-956, 2013.

12. Linn YC and Hui KM: Cytokine-induced NK-like T cells: From bench to bedside. J Biomed Biotechnol 2010: 435745, 2010.

13. Ma YJ, He M, Han JA, Yang L and Ji XY: A clinical study of HBsAg-activated dendritic cells and cytokine-induced killer cells during the treatment for chronic hepatitis B. Scand J Immunol 78: 387-393, 2013

14. Sangha R, Price J and Butts CA: Adjuvant therapy in non-small cell lung cancer: Current and future directions. Oncologist 15 862-872, 2010

15. Severo M, Gaio R, Lourenço P, Alvelos M, Bettencourt $\mathrm{P}$ and Azevedo A: Indirect calibration between clinical observers - application to the New York Heart Association functional classification system. BMC Res Notes 4: 276, 2011

16. Ribeiro JP, Matuguma SE, Cheng S, Herman P, Sakai P, D'Albuquerque LA and Maluf-Filho F: Results of treatment of esophageal variceal hemorrhage with endoscopic injection of n-butyl-2-cyanoacrylate in patients with Child-Pugh class $\mathrm{C}$ cirrhosis. Endosc Int Open 3: E584-E589, 2015.

17. Li Y, Yin J, Li T, Huang S, Yan H, Leavenworth J and Wang X NK cell-based cancer immunotherapy: From basic biology to clinical application. Sci China Life Sci 58: 1233-1245, 2015.

18. Dai H, Wang Y, Lu X and Han W: Chimeric antigen receptors modified T-cells for cancer therapy. J Natl Cancer Inst 108 djv439, 2016

19. Yu D, Du K, Liu T and Chen G: Prognostic value of tumor markers, NSE, CA125 and SCC, in operable NSCLC Patients. Int J Mol Sci 14: 11145-11156, 2013.

20. Wang R, Wang G, Zhang N, Li X and Liu Y: Clinical evaluation and cost-effectiveness analysis of serum tumor markers in lung cancer. Biomed Res Int 2013: 195692, 2013.

21. Pujol JL, Molinier O, Ebert W, Daurès JP, Barlesi F, Buccheri G, Paesmans M, Quoix E, Moro-Sibilot D, Szturmowicz M, et al CYFRA 21-1 is a prognostic determinant in non-small-cell lung cancer: Results of a meta-analysis in 2063 patients. $\mathrm{Br}$ J Cancer 90: 2097-2105, 2004.
22. Cui Y, Yang X, Zhu W, Li J, Wu X and Pang Y: Immune response, clinical outcome and safety of dendritic cell vaccine in combination with cytokine-induced killer cell therapy in cancer patients. Oncol Lett 6: 537-541, 2013.

23. Mitchell DA and Sampson JH: Toward effective immunotherapy for the treatment of malignant brain tumors. Neurotherapeutics 6 : 527-538, 2009.

24. Liu Y, Zhang W, Zhang B, Yin X and Pang Y: DC vaccine therapy combined concurrently with oral capecitabine in metastatic colorectal cancer patients. Hepatogastroenterology 60 : 23-27, 2013

25. Wang M, Shi SB, Qi JL, Tang XY and Tian J: S-1 plus CIK as second-line treatment for advanced pancreatic cancer. Med Oncol 30: 747, 2013

26. Sabado RL and Bhardwaj N: Directing dendritic cell immunotherapy towards successful cancer treatment. Immunotherapy 2 37-56, 2010

27. Mantia-Smaldone GM, Corr B and Chu CS: Immunotherapy in ovarian cancer. Hum Vaccin Immunother 8: 1179-1191, 2012.

28. Minidis NM, Mesner O, Agan BK and Okulicz JF: Delayed-type hypersensitivity (DTH) test anergy does not impact CD4 reconstitution or normalization of DTH responses during antiretroviral therapy. J Int AIDS Soc 17: 18799, 2014.

29. Timmermann C: 'Just give me the best quality of life questionnaire': The Karnofsky scale and the history of quality of life measurements in cancer trials. Chronic Illn 9: 179-190, 2013.

30. Hoos A, Eggermont AM, Janetzki S, Hodi FS, Ibrahim R, Anderson A, Humphrey R, Blumenstein B, Old L and Wolchok J: Improved endpoints for cancer immunotherapy trials. J Natl Cancer Inst 102: 1388-1397, 2010.

31. Pandolfi F, Cianci R, Pagliari D, Casciano F, Bagalà C, Astone A, Landolfi $\mathrm{R}$ and Barone $\mathrm{C}$ : The immune response to tumors as a tool toward immunotherapy. Clin Dev Immunol 2011: 894704, 2011.

32. Yuanying Y, Lizhi N, Feng M, Xiaohua W, Jianying Z, Fei Y, Feng J, Lihua H, Jibing C, Jialiang L and Kecheng X: Therapeutic outcomes of combining cryotherapy, chemotherapy and DC-CIK immunotherapy in the treatment of metastatic non-small cell lung cancer. Cryobiology 67: 235-240, 2013.

33. Ma X, Lin C and Zhen W: Cancer care in China: A general review. Biomed Imaging Interv J 4: e39, 2008.

34. Mellman I, Coukos G and Dranoff G: Cancer immunotherapy comes of age. Nature 480: 480-489, 2011.

35. Kirkwood JM, Butterfield LH, Tarhini AA, Zarour H, Kalinski P and Ferrone S: Immunotherapy of cancer in 2012. CA Cancer J Clin 62: 309-335, 2012.

36. Thomas AA, Ernstoff MS and Fadul CE: Immunotherapy for the treatment of glioblastoma. Cancer J 18: 59-68, 2012.

37. Maggi E: T-cell responses induced by allergen-specific immunotherapy. Clin Exp Immunol 161: 10-18, 2010. 